\title{
Endocarditis infecciosa nosocomial sobre valvula nativa mitral por Staphylococcus lugdunensis
}

\author{
Angel Custodio Sánchez*, Jesús Flores*, José María Martín*, José Antonio Noria*, Antonio Gordi- \\ Ilo*, Benjamín Hernández*, María José Domínguez ** \\ *Unidad de Gestión Clínica de Cuidados Críticos y Urgencias. \\ Hospital Universitario Puerta del Mar. Avenida Ana de Viya 21. 11009. Cádiz. \\ **Unidad de Gestión Clínica de Cuidados Clínicos y Urgencias. \\ Hospital Universitario de Puerto Real. Carretera N-IV Km 665. Cádiz.
}

Se presenta el caso clínico de un paciente de 53 años que tras una intervención neuroquirúrgica (laminectomía) presentó cuadro séptico. Se identificó la presencia de S. lugdunensis en la herida operatoria. El ecocardio- grama transesofágico demostró rotura valvular mitral, vegetaciones e insuficiencia mitral masiva. El paciente se recuperó satisfactoriamente tras reemplazo valvular por prótesis mecánica

\section{Nosocomial mitral valve Endocarditis caused by $S$. lugdunensis}

A 53 year old man developed sepsis following laminectomy for spinal disease. S lugdunensis was identified from the surgical wound. Trans esophageal echocardiography demonstrated mitral valve rupture, vegetations and severe mitral regurgitation. The patient successfully recovered following mitral valve replacement with a mechanical prosthesis

Keywords: S. lugdunensis, Infectious endocarditis, mitral valve replacement 
Introducción: Los estafilococos coagulasa negativos ( $\mathrm{SCoN}$ ) se encuentran entre los microrganismos más frecuentemente aislados en el laboratorio de microbiología. Su significado clínico es difícil de establecer, ya que son comensales de la piel y de las mucosas, y han sido considerados inocuos o patógenos oportunistas con escasa virulencia ${ }^{1}$. La especie Staphylococcus lugdunensis fue descrita por Freney y colaboradores ${ }^{2}$ en 1988. El término lugdunensis deriva de Lugdunum, nombre latino de Lyon, ciudad donde se aisló por primera vez.

S. lugdunensis forma parte de la microbiota de la piel, colonizando principalmente la región perineal. Es un patógeno humano infrecuente y puede causar endocarditis infecciosa sobre válvulas nativas, protésicas o cables de marcapasos. Es frecuente la aparición de abscesos peri valvulares, embolías sépticas e insuficiencia cardiaca. Por ello, es frecuente la necesidad de realizar recambio valvular pre$\mathrm{coz}^{3}$. Este tipo de infecciones puede estar en relación con la capacidad de formar biofilm ${ }^{4-5}$. Aunque pueden causar infecciones en individuos sanos, se ha constatado que los pacientes suelen tener alguna enfermedad de base como diabetes, insuficiencia renal crónica, neoplasias o que reciben terapias inmunosupresoras ${ }^{5}$.

\section{Caso clínico}

Paciente varón de 53 años, con antecedentes de hipertensión arterial, diabetes tipo II en tratamiento con antidiabéticos orales, insuficiencia renal crónica moderada, fumador de 20 cigarrillos/día, cardiopatía isquémica con enfermedad coronaria de dos vasos e insuficiencia mitral leve-moderada por prolapso valvular mitral.

El paciente ingresó en el hospital para ser intervenido de urgencia por el servicio de neurocirugía, debido a la presencia de una estenosis del canal medular secundaria a protrusión discal. Se realizó una laminectomía L3-L4 y hemilaminectomía L5 superior,

A los 15 días del postoperatorio presentó fiebre y disnea, se realizó una ecocardiografía transtorácica que objetivó una insuficiencia mitral severa con jet de $12 \mathrm{~cm}^{2}$ por prolapso del velo anterior mitral y rotura de cuerdas. A pesar del tratamiento (amoxicilina-ácido clavulánico, diuréticos, vasodilatadores y ventilación mecánica no invasiva) el paciente evolucionó desfavorablemente, por lo que ingresó en la unidad de cuidados intensivos (UCI). El cultivo de secreción de la incisión operatoria lumbar demostró la presencia de S. lugdunensis.

Se repitió una ecocardiografía, en esta ocasión transesofá-

Figura 1

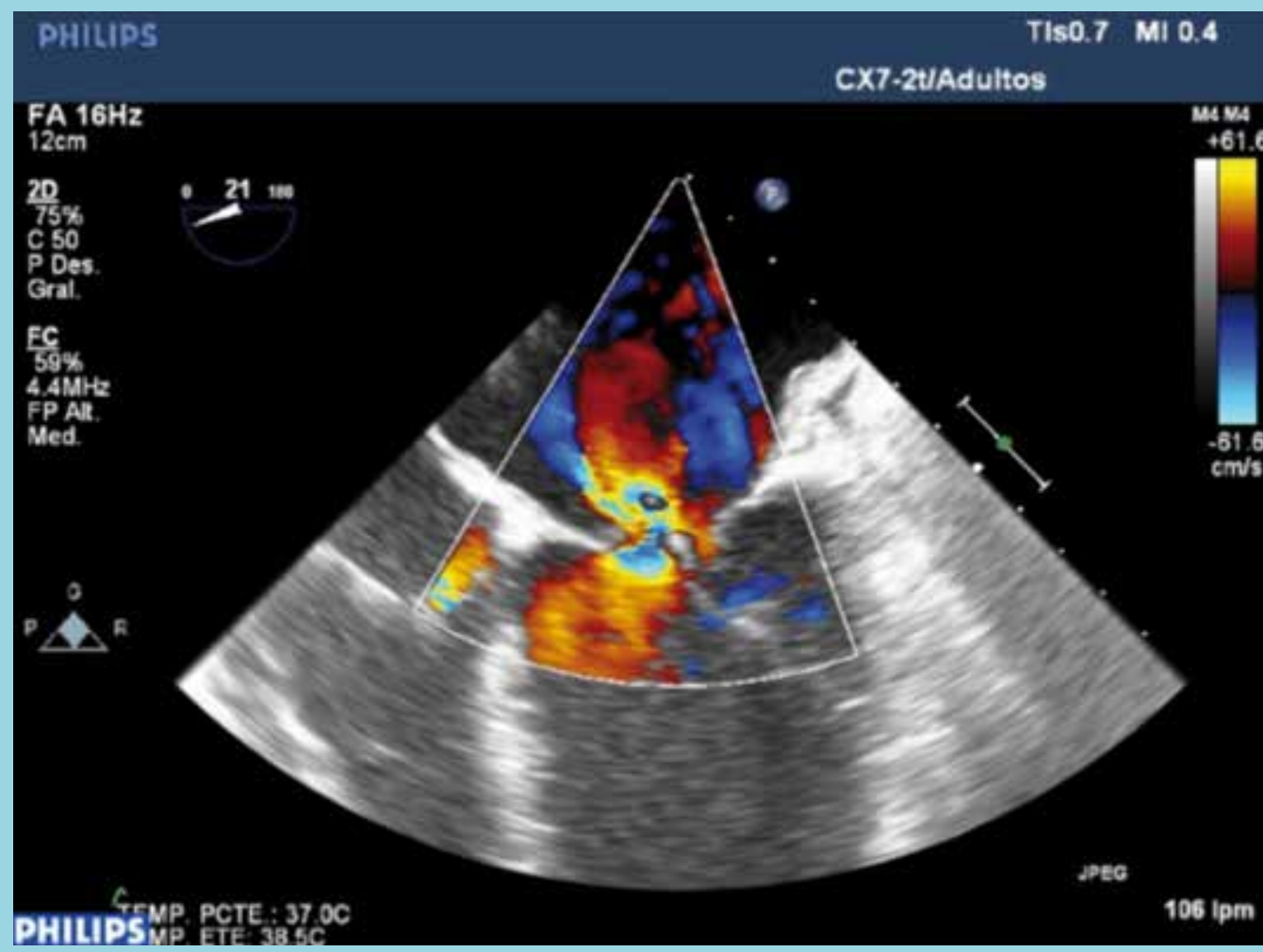




\section{Figura 2.}

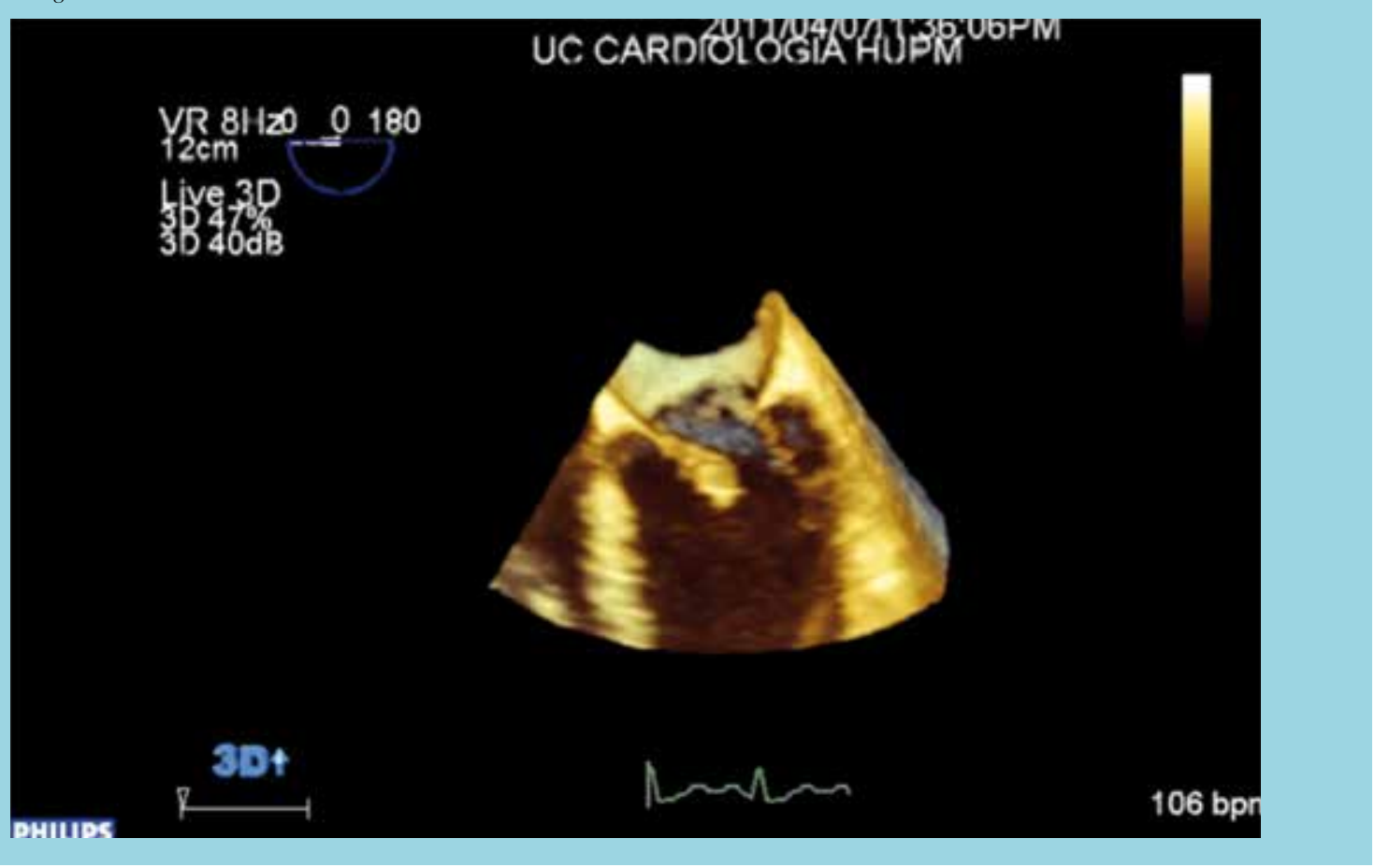

gica, que objetivó una válvula mitral con perforación del velo septal y múltiples vegetaciones móviles, con insuficiencia mitral masiva (Figuras 1 y 2). Se obtuvieron hemocultivos y se inició tratamiento antibiótico con cloxacilina, ampicilina y gentamicina. Se aisló en la herida quirúrgica $\mathrm{y}$ en el hemocultivo el mismo germen (Staphylococcus lugdunensis sensible a penicilina). El paciente necesitó de ventilación mecánica y de perfusión continúa de drogas vaso-activas, continuando con el mismo tratamiento antibiótico. Se realizó cateterismo coronario previo a intervención quirúrgica, implantando dos stents en arteria descendente anterior (Titán) y un stent en arteria marginal proximal (Pro-Kinetic).

Se intervino quirúrgicamente a los 11 días del ingreso en UCI, por insuficiencia cardiaca refractaria, realizando una sustitución valvular mitral por prótesis mecánica. El postoperatorio cursó satisfactoriamente en la UCI, pasando a planta de Rehabilitación a los 10 días de la intervención quirúrgica.

\section{Discusión}

Las infecciones causadas por SCoN, a diferencia de las producidas por Staphylococcus aureus, se manifiestan como enfermedades menos graves o subagudas que raramente se asocian a mortalidad ${ }^{6}$. La excepción a esta regla entre los SCoN es la especie Staphylococcus lugdunensis.
Las características microbiológicas, clínicas y de sensibilidad a antimicrobianos de esta especie la hacen única y diferente de otros SCoN. S. lugdunensis se comporta más como S. Aureus que como un típico SCoN en muchos aspectos, especialmente por su virulencia y por su capacidad para producir infecciones supuradas ${ }^{7}$. Informes de endocarditis infecciosa fulminante y shock séptico dan fe de su virulencia ${ }^{8}$.

S. lugdunensis se ha asociado, con mayor frecuencia que la endocarditis, a un amplio espectro de infecciones, como son las de piel, de tejidos blandos (celulitis, mastitis, abscesos subcutáneos), de huesos y articulaciones (osteomielitis crónica, artritis) y también, como agente causal de bacteriemia, a infecciones asociadas a catéter y a prótesis intravasculares, infecciones del sistema nervioso central, peritonitis asociadas a diálisis peritoneal, infecciones del tracto urinario, endoftalmitis postquirúrgicas, infecciones oculares, óticas, orales e infección del tracto urinario, entre otras $^{6-9}$.

Se debe tener en cuenta, que determinados métodos de laboratorio pueden confundir a S. lugdunensis con otros ECoN o con S. Aureus. S. lugdunensis puede ser confundido con S. Aureus, por su capacidad de producir el factor de afinidad por el fibrinógeno (clumping factor), que constituye un factor de virulencia junto con la capacidad de formación de biofilm ${ }^{4-7}$. S. lugdunensis se diferencia 
del resto de ECoN por producir ornitina descarboxilasa, la prueba de la pirrolidil-arilamidasa y la acidificación de la manosa ${ }^{10-11}$.

En la mayoría de los casos se desconoce la puerta de entrada, mientras que en otros se han señalado las infecciones y las manipulaciones quirúrgicas del área perineal ${ }^{10}$, así como las infecciones del tracto respiratorio inferior ${ }^{11}$. En nuestro caso se demostró infección de herida quirúrgica a nivel lumbar con crecimiento de $S$. lugdunensis en cultivo. La sensibilidad de S. lugdunensis a múltiples clases de antimicrobianos, incluidas las penicilinas, es otra de las características, independientemente del origen del aislamiento, que diferencia a esta especie del resto de los $\mathrm{SCoN}^{7}$. La mayoría de las cepas de S. lugdunensis no producen B-lactamasa, principalmente las de origen europeo ${ }^{7}$.

La bacteriemia por S. lugdunensis se asocia a endocarditis en aproximadamente el $50 \%$ de los pacientes ${ }^{8-12}$. Los pacientes con bacteremia por $S$. lugdunensis adquirida en la comunidad deben ser cuidadosamente examinados en busca de signos de endocarditis. Si la bacteriemia es de origen nosocomial, la endocarditis es menos frecuente, y la bacteriemia por $S$. lugdunensis es más frecuentemente asociada a catéter $u$ otros materiales protésicos ${ }^{12}$. La mayoría de los casos de endocarditis se dan sobre válvulas nativas izquierdas (80\%), se asocian a una mortalidad superior al 38\%, siendo necesaria la intervención quirúrgica precoz para el recambio valvular en la mayoría de los casos $(66,7 \%)$, por la frecuente formación de abscesos, émbolos, destrucción valvular e insuficiencia cardíaca ${ }^{7-13}$. El hallazgo de un cultivo positivo para este germen obliga a la realización de una ecografía transesofágica de forma precoz, para confirmar la presencia de endocarditis. S. lugdunensis nunca debe considerarse como un SCoN contaminante ${ }^{13}$. Las imágenes ecocardiográficas se caracterizan por vegetaciones voluminosas y por la destrucción profunda valvular similar a las producidas por S. aureus ${ }^{14}$.

\section{Conclusiones}

S. lugdunensis es un SCoN atípico en comparación con el resto de los SCoN, tanto por sus características especiales de virulencia como por su llamativa sensibilidad a múltiples antibióticos, incluida en muchos casos la sensibilidad a la penicilina. Su identificación en el laboratorio es sencilla y se debe realizar una identificación completa, principalmente si el germen se aisla de un sitio estéril, ya que rara vez es un contaminante.

Pacientes con bacteriemia por S. lugdunensis, si son adquiridas en la comunidad, deben ser evaluados precozmente mediante una ecografía transesofágica para buscar signos de endocarditis. Una vez que el diagnóstico de endocarditis se confirma, debe considerarse la sustitución valvular de forma precoz, por su elevada mortalidad. 


\section{Referencias:}

1.- HUEBNER J. GOLDMANN DA. Coagulase-negative staphylococci: role as pathogens. Annu Rev Med 1999; 50: 223-36.

2.- FRENEY J, BRUN Y, BES M, MEUGNIER H, GRIMONT F, GRIMONT PAD, et al. Staphylococcus lugdunensis sp. nov. and Staphylococcus schleiferi sp. nov., two species from human clinical specimens. Int J Syst Bacterol. 1998; 38: 168-72.

3.- ANGUERA I, DEL RÍO A, MIRÓ JM, MARTÍNEZ-LACASA X, MARCO F, GUMÁ JR, et al. Staphylococcus lugdunensis infective endocarditis: description of 10 cases and analysis of native valve, prosthetic valve, and pacemaker lead endocarditis clinical profiles. Heart 2005; 91: e10.

4.- MISSINEO A, DI POTO A, GEOGHEGAN JA, RINDI S, HEILBRONNER S, GIANOTTI V, et al. IsdC from Staphylococcus lugdunensis induces biofilm formation under low-iron growth conditions. Infect Immun. 2014; 82: 2448-59.

5.- RODRÍGUEZ-GASCÓN M, ROIG P, MONTAGUD JB, MERINO J. Acute Staphilococcus lugdunensis endocarditis with septic cerebral and pulmonary emboli, showing favorable evolution. Enferm infecc Microbiol Clin 2003; 21:465-7.

6.- FRANK KL, DEL POZO JL, PATEL R. From clinical microbiology to infection pathogenesis: how daring to be different Works for Staphylococcus lugdunensis. Clin Microbiol Rev. 2008; 21:111-33.

7.- CERCENADO E. Staphylococcus lugdunensis: un estafilococo coagulasa negativo diferente a los demás. Enferm Infecc Microbiol Clin. 2009; 27: 139-142.
8.-KLOTCHKO A, WALLACE MR, LICITRA C, SIEGER B. Staphylococcus lugdunensis: An emergeging pathogen. Southern Medical Journal 2011; 104: 509-14.

9.- HELLBACHER C, TORNQVIST E, SODERQUIST B. Staphylococcus lugdunensis: clinical spectrum, atibiotic susceptibility, and phenotypic and genotypic patterns of 39 isolates. Clin Microbiol Infect 2006; 12:43-9.

10.- SÁNCHEZ P, BUEZAS V, MAESTRE JR. Infección por Staphylococcus lugdunensis: presentación de trece casos. Enferm Infecc Microbiol Clin 2001; 19:475-8.

11.- VAN HOOVELS L, DE MUNTER P, COLAERT J, SURMONT M. Mitral and aortic valve endocarditis due to Staphylococcus lugdunensis. Eur J Clin Microbiol Infecc Dis $2005 ; 24: 149-52$.

12.- ZINKERNAGEL AS, ZINKERNAGEL MS, ELZI MV, GENONI M, GUBLER J, ZBINDEN R, et al. Significance of Staphyloccus lugdunensis bacteriemia: report of 28 cases anda review of the literatura. Infection 2008; 36:314-21.

13.- LIU PY, HUANG YF, TANG CW, CHEN YY, HSIEH KS, GER LP, et al. Staphylococcus lugdunensis infective endocarditis: a literatura review and a analysis of risk factors. J Microbiol Immunol Infect 2010; 43:478-84.

14.- SABE MA, SHRESTHA NK, GORDON S, MENON V. Staphylococcus lugdunensis: a rare but destructive cause of coagulase-negative staphylococcus infective endocarditis. Eur Heart J Acute Cardiovasc Care. 2014; 3: 275-80 